Background/introduction Female sex workers (FSWs) are often considered as a vector for HIV and other sexually transmitted infections entering the general communities.

Aim(s)/objectives This study investigated the effectiveness of a resilience-promoting intervention that targets at psychological well-being to facilitate adaptation and safe sexual practices among FSWs which could be an innovative strategy in controlling the spread of these infections.

Methods Using resilience framework, this intervention consisted of six-weekly sessions focused on awareness, expression and management of emotions, identifying roles and personal strengths, and effective problem-solving skills. The primary outcome of resilience and reduction of sexual risk behaviour were assessed at baseline, post-intervention and 3-month follow-ups through self-administered questionnaires. Difference of the differences between the two groups and intention-to-treat analysis were adopted in the analysis.

Results 127 FSWs were recruited and randomly assigned to the intervention or usual care (control) groups in a multi-centred randomised controlled trial. There were significant differences on the score on resilience, self-esteem and general mental health status between the two groups at post-intervention and 3-month follow-ups. The rate of condom use improved with time but significant difference between groups was only observed at 3-month follow-ups. Regression models showed that, after controlling for marital status and family size, intervention group assignment (OR $=2.95,95 \% \mathrm{CI}: 1.19-7.35)$ and self-efficacy $(\mathrm{t}$ $=2.48, \mathrm{p}<0.05)$ was significantly associated with improved resilience scores.

Discussion/conclusion The results suggest that the programme was effective in promoting resilience, self-esteem and the mental health status but with less obvious effect on sexual health among FSWs in Hong Kong.

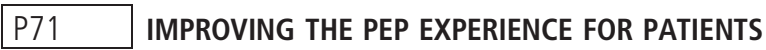

Sarah Mensforth, Lisa Goodall ${ }^{*}$. Stoke and Staffordshire Partnership Trust Sexual Health Department, Stoke on Trent, UK

\subsection{6/sextrans-2015-052126.114}

Background/introduction BHIVA/BASHH have published guidelines with auditable outcomes for initiation of PEPSE and follow up. Some UK centres have, however, reported missing these targets.

Aim(s)/objectives To explore the patient journey from initiation of PEP to completion of follow-up and to identify areas for improvement within our service in supporting patients to take PEP.

Methods Each patient commencing or continuing PEP at our clinic between December 2013 and June 2014 was asked to take part in a survey regarding their experience with PEP. The survey included questions about adherence (motivations and barriers), clinic experience and follow up.

Results 31 patients took PEP during the study period, 26 patients participated in the study. Reasons for PEP included occupational exposure $(\mathrm{n}=6)$, sexual assault $(\mathrm{n}=9)$, and consensual sex $(\mathrm{n}=11) .4$ patients $(15.3 \%)$ reported not completing the 28 day course of PEP. $9(34.6 \%)$ and 8 patients (30.7\%) reported late and missing doses respectively. $88.4 \%$ of patients experienced side effects from medication, only $43 \%$ of patients sought help for this. The most frequent motivation for completing PEP was "fear of HIV infection" (69.2\%). 69.2\% patients identified a specific HCP within clinic as being particularly supportive. Advice regarding remembering to take medication, continuity with HCP and arrangement of follow up appointments at the initial attendance were positively received.

Discussion/conclusion This qualitative survey identified barriers to compliance and ways to support patients in taking PEP. We should encourage patients to contact us for advice regarding side effects and anxiety, and provide practical advice around reminders for medication taking.

\section{P72 INVESTIGATING THE USE OF PRE-EXPOSURE PROPHYLAXIS-A PRELIMINARY ANONYMOUS SURVEY}

Lauren Bull* ${ }^{*}$ Michael Rayment, Joe Phillips, Alan McOwan. Chelsea and Westminster Hospital, London, UK

10.1136/sextrans-2015-052126.115

Background Pre-exposure prophylaxis (PrEP) has been proven to be biologically effective, and its clinical efficacy continues to be assessed in trials. Data have been published on patient's willingness to take PrEP, but we are unaware whether patients are already doing so outside of clinical trials and how they are sourcing the medication.

Methods A preliminary anonymous questionnaire was offered to HIV negative men who have sex with men (MSM) attending our services. The questionnaire sought to gain basic demographic data, the frequency of condomless sex in the preceding three months, whether participants had heard of PreP, whether they had taken it, and if so, how long for and how they sourced the medication.

Results 80 completed questionnaires were returned. The mean age was 30.6 years. The median number of partners in the last three months was 4 (range 1-55). 49\% of respondents had 1-5 episodes of condomless sex in the preceding 3 months and $6 \%$ had $>10$ episodes of condomless sex. The majority of respondents $(82 \%)$ had heard of PrEP. Five respondents (6\%) had taken PrEP: one had been taking part in a clinical trial, three had used post-exposure prophylaxis as PrEP for between 24 and 28 days, and one had used a partner's anti retrovirals for 7 days.

Discussion This preliminary anonymous questionnaire suggests that the majority of HIV negative MSM attending our clinic have knowledge of PrEP. A small number are actively sourcing anti-retroviral medication to use as PrEP outside of clinical trials. We are now expanding this study to reach a higher proportion of our clinic attendees and also to link responses to clinic numbers in order to gain more behavioural information.

\section{P73 THE SOURCE OF THE PROBLEM - RE-AUDIT OF PEPSE PROVISION AT AN INNER CITY SEXUAL HEALTH CLINIC}

Susanna Currie*, Vian Shafiq, Justine Mellor, Jennifer McCord, Jacqueline Houston, Chitra Babu. Manchester Royal Infirmary, Manchester, UK

\subsection{6/sextrans-2015-052126.116}

Background Post exposure prophylaxis following sexual exposure (PEPSE) reduces transmission of HIV. BASHH updated it's guidance on the provision of PEPSE in 2011.

Aim To review provision of PEPSE at an inner city sexual health clinic.

Method Retrospective case note review of patients attending for PEPSE between January and June 2014. Comparison was made 